\title{
Adherence to Universal Precautions and Associated Factors among Nurses Caring For Critically Ill Patients in Dar es Salaam Tanzania
}

Salma A. Wibonela, RN, MSc ${ }^{1 *}$, Columba Mbekenga, RN, $\mathrm{PhD}^{2}$, Fatina B. Ramadhani, RN, $\mathrm{PhD}^{3}$, Ally Mwanga, M.D., M.Med ${ }^{4}$ and Pedro Pallangyo M.D., MPH., M.Med

${ }^{1}$ Nursing Directorate, Jakaya Kikwete Cardiac Institute, Malik Rd, Dar es Salaam, Tanzania

${ }^{2}$ Nursing Critical Care and Trauma, School of Nursing and Midwifery, Aga Khan University, Urambo Street, Dar es Salaam, Tanzania

${ }^{3}$ Department of Clinical Nursing, Muhimbili University of Health and Allied Sciences, United Nations Rd, Dar es Salaam, Tanzania

${ }^{4}$ Surgical Gastroenterologist,School of medicine, Muhimbili University of Health and Allied Sciences, United Nations Rd, Dar es salaam, Tanzania

${ }^{5}$ Research Unit, Jakaya Kikwete Cardiac Institute, Malik Rd, Dar es Salaam, Tanzania

DOI: $10.36348 /$ sjnhc.2020.v03i03.003 | Received: 01.02.2020 | Accepted: 08.02.2020 | Published: 30.03 .2020

*Corresponding author: Salima A Wibonela

Abstract

Background: Globally, it is known that heath care workers particularly in critical care settings are at higher risk of occupational exposure to infections. Surveillance systems to monitor body fluid exposure have been established in developed world. However, such systems are not available and consequently, exposure to body fluids is rarely reported, documented and monitored in many African countries. Objectives: To assess knowledge, practice and factors influencing adherence of Universal Precautions of infection prevention among nurses at Muhimbili national hospital and Muhimbili orthopaedic institute Dar es salaam Tanzania. Design: Quantitative cross-sectional and observational study. SPSS was used for data analysis. Chi-square test and student t-test were used for analysis of categorical and continuous variables respectively and regression analysis were done to determine any significance. Participants: 144 nurses in intensive care units, emergency rooms and recovery rooms were assessed using a structured self-administered questionnaire and an observational checklist to 42 of 144. Findings: $38 \%$ of nurses had good knowledge of universal precautions. $85 \%$ reported to always wash hands, however, $34.3 \%$ of participants were observed to wash hands before putting on gloves. 94.4\% reported to always use gloves, $67.4 \%$ reported to always wear a gown and $55.6 \%$ reported that they always wear a mask. Regarding sharps management $93.8 \%$ reported good practice and $95.2 \%$ demonstrated good sharps management practice. Sex and level of education of an individual significantly influenced the hand hygiene practice, working station and perceived severity significantly influenced the practice of wearing protective gear. Training on infection prevention and control significantly influenced sharps handling (All p<0.05). Discussion: Adherence of universal precautions were sub optimal. Reported and observed practice were incompatible as in other studies except in the practice of sharps management which observed results outweighed the reported results. Conclusion: Adherence to universal precaution is variably poor. Surveillance systems to monitor exposures to body fluids is required.

Keywords: Surveillance systems, infections, analysis, wash hands, body fluids.

Copyright @ 2020: This is an open-access article distributed under the terms of the Creative Commons Attribution license which permits unrestricted use, distribution, and reproduction in any medium for non-commercial use (NonCommercial, or CC-BY-NC) provided the original author and source are credited.

\section{BACKGROUND}

Globally it is well known that healthcare workers and patients especially in critical care settings are more susceptible to contracting infections due to the nature of the critical care environment $[1,2]$.

Frequently close contact with patients, invasive procedures expose them to body fluids and infectious microorganisms.

A systematic review for 21 countries in Africa estimated that about $2 / 3$ of health care workers are exposed to body fluids in their entire career and almost one half are exposed each year [3].

Study done in Ethiopia reported that the lifetime exposure of health care workers to blood and body fluids was $47.4 \%$ [4].

A study conducted in Tumbi and Dodoma hospitals, Tanzania reported the prevalence of occupational exposure to Human immunodeficiency virus (HIV) among health care workers (HCWs) was $47.9 \%[5]$. 
Another study done in Tanzania reported the incidences of percutaneous injuries and mucocutaneous exposure to body fluids among health care workers was about $59 \%$ [6].

Centers for diseases control (CDC) designed safety measures which require (HCW) to comply whenever they come into contact with patients to prevent spread of infections among individuals [7]. They are known as Universal precautions and they are applied to all patient care regardless of suspected or confirmed infection status. Such measures include; hand hygiene using either soap and water or disinfectant solution (hand rub), use of personal protective equipment depending on the anticipated exposure, safe injection practice and sharps management, proper decontamination procedures and good environmental hygiene.

With the evidence of high prevalence of exposure to body fluids in critical care setting, it is crucial for health care workers working in these settings to adhere to universal precautions to reduce the risk of spreading infections among themselves and with patients. Unfortunately, different studies show sub optimal adherence to universal precautions among health care workers [8-10]. This creates many questions as to whether health care providers understand universal precaution measures of infection prevention and control.

The 2012 Tanzania National Guideline for HIV Prevention and the 2014 National audit office through the Ministry of health and social welfare emphasize on the implementation of universal precautions for infection prevention $[11,12]$.

Hence this study intended to assess knowledge, practice and factors that influence adherence to universal precautions for infection prevention and control among nurses caring for critically ill patients at Muhimbili national hospital and Muhimbili Orthopaedic Institute, Dar es Salaam, Tanzania.

\section{METHOD}

This was a quantitative cross sectional and observational study.

\section{Population}

All trained nurses working in Intensive Care Units, Emergency rooms and Recovery rooms at Muhimbili National Hospital and Muhimbili Orthopaedic Institute in Dar Es Salaam, Tanzania. Muhimbili National Hospital had a total of 177 nurses and Muhimbili Orthopaedic Institute had 64 nurses in these critical care settings. Sample size estimation was done using finite population formula, 144 nurses were recruited for study using stratified simple random sampling. All nurses working in Critical care settings in
ICU, emergence rooms and recovery rooms and directly involved in patient care were eligible to participate in this study.

\section{Data Collection Tools}

A structured self-administered questionnaire and an observational checklist were used to collect data. The tools were adopted from study which was done in Ethiopia by Gebresilassie et al., assessing standard precautions practice among health care workers in public health facilities [10]. The tools were modified with regard to study area. A pilot study was done among 20 nurses [13] and modification of questionnaire was done accordingly. The questionnaire had four parts; demographic data, knowledge assessment, practice assessment and factors for not practicing universal precautions for infection prevention and control.

\section{Procedure}

The researcher obtained informed consent from study participants followed by administration of the questionnaire to each participant $(\mathrm{N}=144)$. The observation part of data collection was done among 42 out of 144 nurses with the aim of obtaining practice data. The observation started one week later after introduction to the study participants in order to minimize the hawthorn effect. Also delaying was assumed to help the researcher and research assistants to get familiarity with the environment. The type of observation was "participant observant". Where by a researcher studied participants by observing and participating in practicing [14] Data collection was done during the morning and evening shifts. Filling of questionnaire was done later after observation for those nurses whom were observed. Observation took 45 minutes and filling of questionnaire took 30 minutes. Data collection was done from march to April 2015.

Knowledge on blood borne viruses and its mode of transmission among study participants was assessed using two structured questions with a total of eight scores [8] correct responses. It was then categorized into two groups; good knowledge was categorized to those health care workers who responded well from 5-8 correct responses and those health care workers who responded well from 0-4 correct responses were categorized as they had poor knowledge.

Knowledge on Prevention (universal precaution) was assessed using four structured questions with total of 18 scores, it was then categorized into two groups; those HCWs who responded well to 9-18 correct responses were categorized as they have good knowledge and those who responded well from 0-8 correct responses [15].

Reported compliance to universal precautions was categorized as "good" only when the study participant responded consistently (always) for the six structured questions that addressed compliance to 
Universal precautions (hand hygiene, proper sharps handling and use of personal protective equipment) [10]. While from observation data compliance was categorized as "good" only when an observed participant:

- Performed hand hygiene, by observing the hand hygiene moments

- Weared PPEs: for procedures likely to come into contact with body fluids, procedures likely to generate splashes or droplets of body fluids

- Did not recap needle after use.

\section{DATA ANALYSIS}

The data was analyzed using SPSS version 16. Chi Square tests $\left(\mathrm{X}^{2}\right)$ for comparison of proportions among categorical variables student t-test was used for analysis of continuous variables and ODDs ratio (OR) were used in analysis to determine any statistical significance

\section{Ethical Consideration}

The researcher got ethical clearance from the Institutional Review Board (IRB) of Muhimbili University of Health and Allied Sciences prior to the study. As this study was a dissertation for accomplishment of Master's degree. Permission to conduct the study was obtained from MNH and MOI research committee. Also, permission to conduct a pilot study was obtained from Ilala Municipality. Participants were assured of anonymity and participation in the study (whereby aim of the study was well explained to them, confidentiality and privacy of the study participants was observed.

\section{Findings}

This study intended to assess knowledge, practice and factors that influence adherence to universal precautions for infection prevention and control among nurses.

\section{Participant Characteristics}

Majority $121(84 \%)$ of 144 participants were females. Their mean age was 35.7 years More than half (56.3\%) had Diploma level of education and $69.4 \%$ attained training on Infection prevention and control.

97.2\% of participants revealed high perceived severity, meaning they perceive that the consequences after contracting blood borne diseases are very bad.

$12.5 \%$ of participants revealed they were exposed to splashes of body fluids and blood in the past one year.

$15.3 \%$ revealed they were exposed to needle stick injuries in the past one year.

\section{Knowledge}

Concerning knowledge on blood borne viruses and their mode of transmission, $74.3 \%$ of participants had good knowledge.

With regards to knowledge on prevention (universal precautions) only $38 \%$ revealed good knowledge.

\section{REPORTED PRACTICE}

$85.4 \% \quad(n=144)$ of the study participants reported that they observe good compliance to hand hygiene.

93.8\% $(n=144)$ of the study participants reported that they observe good compliance to sharps handling.

$94.4 \% \quad(n=144)$ of the study participants reported that they observe good compliance to wearing gloves.

$55.6 \%(n=144)$ of the study participants reported that they observe good compliance to wearing mask/goggles.

$67.4 \% \quad(n=144)$ of the study participants reported that they observe good compliance to wearing gown/apron.

\section{Observed Practice}

Among the 10 rooms which were observed only 6 rooms had running water closer to patient care area. Also only six rooms had posters and pictures for risk communication.

Observed results of hand hygiene was as follows;

$11 / 38(34.3 \%)$ were observed to comply to hand hygiene before wearing gloves 14/38(36.8\%) were observed to comply to hand hygiene after removal of gloves.

Sharps handling; Among the 42 observed participants $2(4.8 \%)$ recapped needles and 40(95.2\%) did not reccaped needles after use.

Use of personal protective equipment; 33/42(78.6\%) weared gloves during parenteral medications, $13 / 26(50 \%)$ weared mask during suctioning respiratory secretions and 11/15(73.3\%) weared gown/apron during bed bath

\section{Factors That Influence Universal Precautions Practice}

The odd of good compliance to hand hygiene was 9 times higher in female compared to male nurses (AOR $8.8 ; 95 \%$ CI $2.05-38.14$ ) 
The odd of good compliance to hand hygiene was 6 times higher among those with the education level of degree and above compared to those with certificate level (AOR 5.9; 95\% CI 1.0 - 34.0).

The odd of good sharps handling (never recapping of needles) was likely to be reduced by $18 \%$ among those who were not attained training on infection prevention and universal precautions compared to those who attained training (AOR 0.18; $95 \%$ CI $0.04-0.84)$.
Compared to participants who were working in ICU, those participants who were working in EMD and recovery room had $71 \%$ and $45 \%$ reduced odd of good practice of wearing gown/apron for procedures likely to generate splashes of blood and body fluids (AOR 0.07; 95\% CI $0.009-0.6$ ) and (AOR 0.05; 95\% CI $0.005-$ 0.37 ) respectively.

The odds of good practice of wearing gloves was 35 times more among those participants with high perceived severity compared to those with low perceived severity (AOR 35; 95\% CI 1.816-655.4).

Table-1: Demographics of the participants

\begin{tabular}{|c|c|c|c|}
\hline Variables & Frequency & Mean & STD deviation \\
\hline \multicolumn{4}{|l|}{ Age groups } \\
\hline $18-35 y r s$ & $81(56.3 \%)$ & \multirow[t]{2}{*}{35.71} & \multirow[t]{2}{*}{8.79} \\
\hline $35-57 \mathrm{yrs}$ & $63(43.8 \%)$ & & \\
\hline \multicolumn{4}{|l|}{ Sex } \\
\hline Male & $23(16.0 \%)$ & & \\
\hline Female & $121(84.0 \%)$ & & \\
\hline \multicolumn{4}{|l|}{ Level of education } \\
\hline Certificate & $24(16.7 \%)$ & & \\
\hline Diploma & $81(56.3 \%)$ & & \\
\hline Degree and above & $39(27.1 \%)$ & & \\
\hline \multicolumn{4}{|l|}{ Experience } \\
\hline$<5$ & $58(40.3 \%)$ & \multirow[t]{3}{*}{1.80} & \multirow[t]{3}{*}{.753} \\
\hline $5-15$ & $57(39.6 \%)$ & & \\
\hline $15+$ & $29(20.1 \%)$ & & \\
\hline \multicolumn{4}{|l|}{ Work station } \\
\hline \multicolumn{4}{|l|}{ MNH } \\
\hline ICU & $58(40.3 \%)$ & & \\
\hline RROOM & $17(11.8 \%)$ & & \\
\hline EMD & $35(24.3 \%)$ & & \\
\hline \multicolumn{4}{|l|}{ MOI } \\
\hline ICU & $11(7.6 \%)$ & & \\
\hline RROOM & $9(6.3 \%)$ & & \\
\hline EMD & $14(9.7 \%)$ & & \\
\hline \multicolumn{4}{|c|}{ Training on Universal Precaution } \\
\hline Yes & $100(69.4 \%)$ & & \\
\hline No & $44(30.6 \%)$ & & \\
\hline \multicolumn{4}{|c|}{ Previous exposure to needle stick } \\
\hline Yes & $22(15.3 \%)$ & & \\
\hline No & $122(84.7 \%)$ & & \\
\hline \multicolumn{4}{|c|}{ Previous exposure to splashes } \\
\hline Yes & $18(12.5 \%)$ & & \\
\hline No & $126(87.5 \%)$ & & \\
\hline
\end{tabular}

Majority $121(84 \%)$ of 144 participants were females. Their mean age was 35.7 yrs, Almost more than half $(56.3 \%)$ had Diploma level and $69.4 \%$ attained training.

Table-2: Reported compliance to Universal Precaution (n=144)

\begin{tabular}{|l|l|l|}
\hline \multicolumn{3}{|l|}{ Practice } \\
\hline Variable & Good practice & Poor practice \\
\hline Hand hygiene & $123(85.4 \%)$ & $21(14.6 \%)$ \\
\hline Sharps handling & $135(93.8)$ & $9(6.3 \%)$ \\
\hline Use of PPE & \multicolumn{2}{|l|}{} \\
\hline Gloves & $136(94.4 \%)$ & $8(5.6 \%)$ \\
\hline Masks/goggle & $80(55.6 \%)$ & $64(44.4 \%)$ \\
\hline Gown/apron & $97(67.4 \%)$ & $47(32.6)$ \\
\hline
\end{tabular}


Table-2 above show the following;

$85.4 \%$ reported good adherence to hand hygiene.

Further question was asked for those who reported poor adherence reasons and $38 \%$ and $23.8 \%$ said "it increases patient waiting time and not all patient contact is infectious" respectively With regard to use of
PPE most participants reported good practice of using gloves compared to mask and gown/apron.

For those who revealed poor practice of wearing personal protective equipment the prevail reason for noncompliance was "lack of supply" Factors affecting Compliance to Universal Precautions among study participants $(n=144)$.

Table-3: Factors affecting compliance to universal precautions

\begin{tabular}{|c|c|c|c|c|c|}
\hline \multirow[t]{2}{*}{ Practice } & \multirow[t]{2}{*}{ Variables } & \multirow[t]{2}{*}{ AOR } & \multicolumn{2}{|c|}{$\begin{array}{l}\text { 95.0\% C.I. for } \\
\text { AOR }\end{array}$} & \multirow[t]{2}{*}{$P$-Value } \\
\hline & & & Lower & Upper & \\
\hline \multicolumn{6}{|c|}{ Hand hygiene } \\
\hline & Sex & 8.834 & 2.046 & 38.139 & 0.004 \\
\hline & Level of education & & & & \\
\hline & Degree and above & 5.865 & 1.013 & 33.961 & 0.048 \\
\hline \multicolumn{6}{|c|}{ Sharps handling } \\
\hline & Training on IPC & .175 & .036 & .842 & 0.030 \\
\hline \multicolumn{6}{|c|}{ Use of PPE } \\
\hline Gloves & Perceived severity & 34.501 & 1.816 & 655.374 & 0.018 \\
\hline Gown & \multicolumn{5}{|l|}{ Work station } \\
\hline & EMD & .071 & .009 & .573 & 0.013 \\
\hline & Recovery room & .045 & .005 & .369 & 0.004 \\
\hline
\end{tabular}

Reference variables: 35 and above, male, certificate, 11years and above, ICU, low perception, low susceptibility, trained.

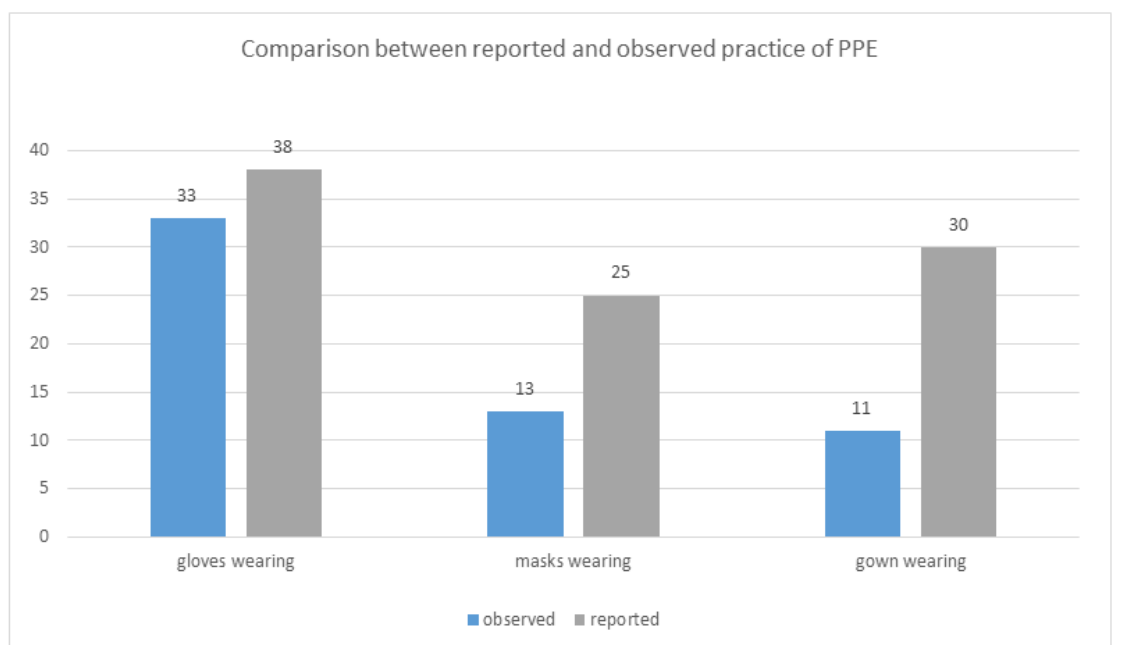

Fig-1: Comparison of the respondent in reported and observed Compliance of PPE

The Figure-1 above shows that the reported compliance to PPE use is higher compared to observed.

\section{DISCUSSION}

Health care workers and patients may be prevented from infections by adhering to universal precaution principles [7]. Unfortunately, many researches worldwide show adherence among health care workers is low, this study also revealed the same.
In this study more than half of the study participants $(69 \%)$ reported they attained training on infection prevention and universal precautions. This relate to another study which was done in Tanzania assessing knowledge of occupational exposure to HIV, it showed that over $70 \%$ of participants had got training on use of personal protective equipment and on infection prevention and control [16]. Concerning knowledge on universal precautions this study revealed only $38 \%$ had good knowledge. This is similar to the study done in Nigeria which revealed knowledge level 
of $38.8 \%$ [17]. The results differ from another study done in Tanzania which showed that $87 \%$ of participants were aware of the concepts of universal precautions [18]. The percentage seem to be a bit higher compared to that from Kenya which showed that only $17.8 \%$ had shown a good knowledge [9]. Nurses were well informed on personal protective equipment as high percent mentioned them, similar to study done in Tumbi and Dodoma Tanzania by [16]. However $52.4 \%$ of participants did not know that personal protective equipment are to be worn depending on expected exposure and not all the personal protective equipment to be worn in every procedure and all the time.

\section{Practice of Universal Precautions}

With regard to hand hygiene as it is recommended to comply to moments of hand hygiene $85.4 \%$ of participants reported they always wash hands for any direct contact with patients in observation only $34.3 \%$ actually performed hand hygiene before putting on gloves. The study done in Kenya showed reported compliance of $87.8 \%$ and in observation was $33.3 \%$ [9]. In Ethiopia it showed $61.5 \%$ and observed practice was $36 \%$. However the results differ from study done in Nigeria which revealed that $82.1 \%$ washed their hands before touching patients [19].

In this study females were more likely to comply to hand hygiene than males and the difference was statistically significant $(\mathrm{p}=0.04)$. This was same as what was seen in Kenya, Ethiopia and Nigeria by [9, $10,8]$ respectively where by females were more compliant than males. The reason could be male perceive themselves as strong they cannot get infected easily [8]. Also, those who attained level of education of degree and above were more likely to comply to hand hygiene than those with diploma and certificate $(p=0.048)$. The possible reason could be there is improved curriculum for infection prevention and control particularly hand hygiene in higher learning.

The major reported reason for poor compliance among those who reported not to comply to hand hygiene was "it is waste of time and increases patient waiting time" Concerning use of personal protective equipment; This study revealed that $94.4 \%$ of participants reported that they always used gloves, $56 \%$ always used mask and $67.4 \%$ always used gown. In other studies, it showed $95.6 \%$ utilized gloves, $61.1 \%$ mouth nose and eye protector and $92.2 \%$ utilized gown [9]. In Bengal India it showed wearing gloves, apron and goggles was $62.4 \%, 56.2 \%$ and 22.5 respectively [20]. Compliance to wearing gloves prevailed in many studies compared to other personal protective equipment as seen in $[10,9,20,21]$.

This study revealed that those participants who had high perceived severity were more likely to observe good practice of wearing gloves compared to those with low perceived severity and the difference was statistically significant ( $\mathrm{p}=0.018$ ) According to health belief theory individual perception on the consequences which can be resulted due to exposure to blood borne viruses may create perceived threat and hence may lead to adherence to preventive measures [22].

The odd of good compliance of wearing gown were likely to be reduced among participants who were working in emergency rooms and recovery rooms compared to those whom were working in ICU and the difference was statistically significant $(p=0.013)$ and $(p=0.004)$ respectively. This might be due to the nature of working environment where by in emergency rooms patients comes abruptly and needs quick management so nurses may weigh between patient life and protective gear [8].

Majority of the respondents who showed poor compliance to personal protective equipment reported that the major reason for non compliance was "lack of availability" other studies also reveal the same [10,9].

In this study the observed compliance in hand hygiene practice and use of personal protective equipment seem to be much lower compared to the reported compliance. Other studies reveal the same [10, 9].

The reason for discrepancy could be social desirability bias that individuals exaggerates their reported compliance than the actual [10]. According to systematic review of studies on compliance with hand hygiene guidelines in hospital care, it showed that approximately $90 \%$ of all studies which were reviewed used direct observation and it was concluded that direct observation was norm when it comes to measuring compliance [23].

With regard to handling sharps this study showed that $92.8 \%$ reported that they did not recap needles and in observation it was revealed that $95.2 \%$ did not recap needles. This seem to relate with study done in Kuwait which showed $92.3 \%$ of participants had good practice of sharps management [24].

The reason of improved sharps management could be facilitated by proper training on sharps handling. In this study the odd of good practice of handling sharps was likely to be higher in nurses who trained in infection prevention and control than those who did not take training and the difference was statistically significant ( $\mathrm{p}=0.03$ ) This is similar to study done in Ethiopia and Kenya by $[9,10]$. However it seem to be different from another study which was done in Ethiopia in which it showed training was not a predictor of good compliance [25]. Other studies show that knowledge only is not a predictor of translation to practice there might be other factors [20]. 
Implementing a training package may help but systems need to be in place as well such as availability of constant running water and antimicrobial hand rubs, availability of personal protective equipment, adequate staff, training and supervision, all these together would facilitate adherence with universal precautions [21].

A qualitative study done in Nigeria assessing psychosocial and organizational factors relating to adherence to standard precautions showed there are three factors influence adherence include individual, work related and organizational that is, first healthcare worker with his or her personal characteristics and professional experience, second is type of tasks and dynamics of the healthcare work, in which the care demands may compete with personal safety, and the third is organizational context, in which safety may have a cultural value and the management may support the use of standard precautions [26]

Limitation of this study is possibility of recall bias which might resulted in misclassification of practice. This due to retrospective assessment of occupational exposure to blood and body fluids [21].

\section{CONCLUSION}

Adherence to Universal precaution seems to be a problem in the study setting. The overall knowledge on universal precaution is poor. The reported compliance to hand hygiene and uses of personal protective equipment is high compared to observed compliance. Sex and level of education of an individual was found to be a significant determinant of hand hygiene practice. Working area and perceived severity has found to be a significant determinant of use of personal protective equipment. Training on infection prevention and control have found to have significant effect on sharps handling.

\section{RECOMMENDATION}

To ensure provision of water supply near patient care area and antimicrobial hand rubs to all patient bedside. Ensure adequate supply of personal protective equipment and other equipment for IPC. Provision of continuous training to nurses on infection prevention and control. Creating systems of surveillance and continuous feedback to health care. Qualitative study needs to be conducted to explore more factors associated with adherence to universal precaution measures.

Improve awareness of occupational exposure to blood borne diseases Curriculum for ipc to put enogh emphasis on prevention.

\section{REFERENCES}

1. Smith, H., Brooks, J. E., Leaptrot, D., AllenBridson, K., Anttila, A., Gross, C., ... \& Wright, M. O. (2017). Health care-associated infections studies project: An American Journal of Infection
Control and National Healthcare Safety Network data quality collaboration. American journal of infection control, 45(6), 612-614.

2. Elston, J., Hinitt, I., Batson, S., Noakes, C., Wright, J., Walley, J., \& Humphreys, C. (2013). Infection control in a developing world. Health estate, 67(10), 45-50.

3. Auta, A., Adewuyi, E. O., Tor-Anyiin, A., Aziz, D., Ogbole, E., Ogbonna, B. O., \& Adeloye, D. (2017). Health-care workers' occupational exposures to body fluids in 21 countries in Africa: systematic review and meta-analysis. Bulletin of the World Health Organization, 95(12), 831.

4. Amerga, E. W., \& Mekonnen, T. G. (2018). Occupational Exposure to Blood and Body Fluids among Health Care Workers in Arada Sub-city Health Centers of Addis Ababa, Ethiopia. Occup Med Health Aff, 6(281), 2.

5. Mashoto, K. O., Mubyazi, G. M., Mohamed, H., \& Malebo, H. M. (2013). Self-reported occupational exposure to HIV and factors influencing its management practice: a study of healthcare workers in Tumbi and Dodoma Hospitals, Tanzania. BMC health services research, 13(1), 276.

6. Laisser, R. M., \& Ng'home, J. F. (2017). Reported incidences and factors associated with percutaneous injuries and splash exposures among healthcare workers in Kahama District, Tanzania. Tanzania Journal of Health Research, 19(1).

7. Roup, B. J. (1997). Factors associated with compliance of critical care nurses with universal precautions: a pilot study. American Journal of Critical Care, 6(3), 218-224.

8. Efstathiou, G., Papastavrou, E., Raftopoulos, V., \& Merkouris, A. (2011). Factors influencing nurses' compliance with Standard Precautions in order to avoid occupational exposure to microorganisms: A focus group study. BMC nursing, 10(1), 1.

9. Moyo, G. M. (2013). Factors influencing compliance with infection prevention standard precautions among nurses working at Mbagathi district hospital. Nairobi, Kenya.

10. Gebresilassie, A., Kumei, A., \& Yemane, D. (2014). Standard precautions practice among health care workers in public health facilities of Mekelle special zone, Northern Ethiopia. J Community Med Health Educ, 4(3), 286.

11. National-Aids-Control-Programme-(NACP). (2012). The United Republic of Tanzania National Guidelines for the management of HIV and AIDS. 4th ed. Dar es Salaam, Tanzania: Ministry of Health and Social Welfare; 304.

12. Tanzania-National-Audit-Office. (2014). The United Republic of Tanzania: Performance audit on the management of healthcare waste. Dar es Salaam, Tanzania: Ministry of Health and Social Welfare; 109.

13. Johanson, G. A., \& Brooks, G. P. (2010). Initial 
scale development: sample size for pilot studies. Educational and psychological measurement, 70(3), 394-400.

14. Research Design.

15. Janjua, N. Z., Razaq, M., Chandir, S., Rozi, S., \& Mahmood, B. (2007). Poor knowledge-predictor of nonadherence to universal precautions for blood borne pathogens at first level care facilities in Pakistan. BMC infectious diseases, 7(1), 81.

16. Mashoto, K. O., Mubyazi, G. M., \& Mushi, A. K. (2015). Knowledge of occupational exposure to HIV: a cross sectional study of healthcare workers in Tumbi and Dodoma hospitals, Tanzania. BMC health services research, 15(1), 29.

17. Bamigboye, A. P., \& Adesanya, A. T. (2006). Knowledge and Practice of Universal Precautions among Qualifying Medical and Nursing Students: A Case of Obafemi Awolowo University Teaching Hospitals Complex, ILEIFE. Res J Med Med Sci, 1(3), 112-116.

18. Chalya, G., Chalya, P. L., \& Mbunda, F. (2016). Knowledge, practice and factors associated with poor compliance with universal precautions among healthcare workers at Bugando Medical Centre, Mwanza, Tanzania. Tanzania Journal of Health Research, 18(3).

19. Abdulsalam, M., Ibrahim, A., Michael, G., \& Mijinyawa, A. (2015). Hand washing practices and techniques among health professionals in a tertiary hospital in Kano. Journal of Medical Investigations and Practice, 10(1), 8.

20. Mukherjee, S., Bhattacharyya, A., SharmaSarkar,
B., Goswami, D. N., Ghosh, S., \& Samanta, A. (2013). Knowledge and practice of standard precautions and awareness regarding postexposure prophylaxis for HIV among interns of a medical college in West Bengal, India. Oman medical journal, 28(2), 141-145.

21. Amoran, O. E., \& Onwube, O. O. (2013). Infection control and practice of standard precautions among healthcare workers in northern Nigeria. Journal of global infectious diseases, 5(4), 156-163.

22. Abraham, C. (2015). The Health Belief Model.

23. Erasmus, V., Daha, T. J., Brug, H., Richardus, J. H., Behrendt, M. D., Vos, M. C., \& van Beeck, E. F. (2010). Systematic review of studies on compliance with hand hygiene guidelines in hospital care. Infection Control \& Hospital Epidemiology, 31(3), 283-294.

24. Karim, J., Al-Saraji, M., Al-Mousawi, F., AlHaddad, Z., Al-Sharaf, D., Marwan, Y., \& Akhtar, S. (2012). Knowledge and self-reported practice of universal precautions among Kuwait University medical students in their clinical years. Medical Principles and Practice, 21(4), 328-333.

25. Reda, A. A., Fisseha, S., Mengistie, B., \& Vandeweerd, J. M. (2010). Standard precautions: occupational exposure and behavior of health care workers in Ethiopia. PLoS One, 5(12):e14420.

26. Brevidelli, M. M., \& Cianciarullo, T. I. (2009). Psychosocial and organizational factors relating to adherence to standard precautions. Revista de saude publica, 43, 907-916. 